\title{
Idiopathic intracranial hypertension: 120-day clinical, radiological, and manometric outcomes after stent insertion into the dural venous sinus
}

\author{
*Hasan Asif, MRCS, Claudia L. Craven, MSc, MRCS, Almas H. Siddiqui, MBBS, \\ Syed N. Shah, MBBS, Samir A. Matloob, MRCS, Lewis Thorne, FRCS(SN), \\ Fergus Robertson, MD, MA, MRCP, FRCR, Laurence D. Watkins, MA, FRCS(SN), and \\ Ahmed K. Toma, MD, FRCS(SN) \\ Victor Horsley Department of Neurosurgery, National Hospital for Neurology and Neurosurgery, London, United Kingdom
}

OBJECTIVE Idiopathic intracranial hypertension (IIH) is commonly associated with venous sinus stenosis. In recent years, transvenous dural venous sinus stent (DVSS) insertion has emerged as a potential therapy for resistant cases. However, there remains considerable uncertainty over the safety and efficacy of this procedure, in particular the incidence of intraprocedural and delayed complications and in the longevity of sinus patency, pressure gradient obliteration, and therapeutic clinical outcome. The aim of this study was to determine clinical, radiological, and manometric outcomes at 3-4 months after DVSS in this treated IIH cohort.

METHODS Clinical, radiographic, and manometric data before and 3-4 months after DVSS were reviewed in this single-center case series. All venographic and manometric procedures were performed under local anesthesia with the patient supine.

RESULTS Forty-one patients underwent DVSS venography/manometry within 120 days. Sinus pressure reduction of between 11 and $15 \mathrm{~mm} \mathrm{Hg}$ was achieved 3-4 months after DVSS compared with pre-stent baseline, regardless of whether the procedure was primary or secondary (after shunt surgery). Radiographic obliteration of anatomical stenosis correlating with reduction in pressure gradients was observed. The complication rate after DVSS was $4.9 \%$ and stent survival was $87.8 \%$ at 120 days. At least $20 \%$ of patients developed restenosis following DVSS and only $63.3 \%$ demonstrated an improvement or resolution of papilledema.

CONCLUSIONS Reduced venous sinus pressures were observed at 120 days after the procedure. DVSS showed lower complication rates than shunts, but the clinical outcome data were less convincing. To definitively compare the outcomes between DVSS and shunts in $\mathrm{IH}$, a randomized prospective study is needed.

https://thejns.org/doi/abs/10.3171/2017.4.JNS162871

KEY WORDS endovascular; dural venous sinus stenting; venous sinus pressure; intracranial pressure; cerebrospinal fluid; idiopathic intracranial hypertension; interventional neurosurgery

I DIOPATHIC intracranial hypertension (IIH) affects 1-3 per 100,000 patients and presents clinically with disabling headaches, tinnitus, and risk of blindness. ${ }^{15}$ It is characterized by raised intracranial pressure (ICP) and elevated CSF opening pressure on lumbar puncture with normal CSF composition in the absence of hydrocephalus and CNS space-occupying lesions. ${ }^{19}$
Despite being first described in the late 19th century and more common in the obese and women of childbearing age, the pathophysiology behind IIH remains unclear. ${ }^{3,12,15}$ IIH is initially managed medically with weight loss and ICP-lowering drugs. When medication fails, surgical management options include CSF diversion, which is typically shunt insertion, or optic nerve sheath fenestration. How-

ABBREVIATIONS DVSS = dural venous sinus stent; ICP = intracranial pressure; $I I \mathrm{H}=$ idiopathic intracranial hypertension; $\mathrm{LP}=$ lumboperitoneal; $S S S=$ superior sagittal sinus; $V P=$ ventriculoperitoneal.

SUBMITTED November 16, 2016. ACCEPTED April 4, 2017.

INCLUDE WHEN CITING Published online October 6, 2017; DOI: 10.3171/2017.4.JNS162871.

* Drs. Asif and Craven contributed equally to this work. 
ever, surgical approaches are not benign and involve their own set of risks, and multiple surgical revisions for IIH are normal..$^{10}$ Furthermore, narrow lateral ventricles combined with increased tissue adiposity can make shunt surgery more complex, with potential difficulty in adjusting valves and increased shunt failure rates. ${ }^{1}$

Several interacting causative mechanisms have been postulated in IIH, including venous hypertension and impaired CSF absorption. ${ }^{4}$ Imaging studies often demonstrate venous sinus narrowing, either from intrinsic intraluminal impediments such as prominent arachnoid granulations, or from extrinsic compression from swollen brain tissue. ${ }^{23}$ Endovascular obliteration of these narrowings with dural venous sinus stenting (DVSS) has therefore emerged as a minimally invasive alternative to surgery in resistant cases of $\mathrm{IIH}^{7}$

Although early clinical improvement following stent insertion has been described, sustained obliteration of anatomical stenosis, manometric gradient, control of ICP, and symptomatic improvement remain unproven. Furthermore, the causal relationship between radiographic anatomical stenosis and raised ICP remains unclear. ${ }^{3,23} \mathrm{~A}$ recent Cochrane review suggested neither stent insertion nor CSF diversion as a preferential technique. Stent placement remains a contentious first-line procedure for IIH..$^{13}$

In the authors' unit, all patients with clinicoradiological diagnosis of IIH resistant to conventional medical therapy are offered continuous ICP monitoring and formal catheter cerebral venography with pressure measurements. If a stenosis with a significant pressure gradient is demonstrated, then patients are counseled and offered the option for DVSS as an alternative to the standard therapy of surgical CSF diversion. All patients treated with endovascular stenting undergo routine clinical review and repeat venography with pressure measurements at 3-4 months after the procedure.

In this study we review the clinical, radiological, and manometric outcomes in patients with IIH and the efficacy of stent insertion (as determined by the requirement for a further procedure) at 3-4 months follow-up.

\section{Methods}

\section{Study Design and Participants}

We reviewed a single-center retrospective case series of patients with a diagnosis of IIH who underwent DVSS between September 2010 and March 2016. Demographic data included age, sex, and previous operative or medical management for raised ICP. Two patient groups were defined: 1) primary, DVSS as a first-line procedure; and 2) secondary, DVSS as a second-line procedure. All patients consented to the stent procedure. Ethical approval was not required for this retrospective study of current practice.

\section{Clinical Data}

Clinical data including patient-reported symptoms and clinical signs were retrospectively collected from standard neurosurgery and neuroophthalmology assessments at the two time points: presenting, and at 3-4 months after stent placement.

\section{Venography and Manometry}

The authors found cross-sectional imaging (including CT/MR venography) unreliable in identifying patients with manometric stenosis, and therefore this was principally used to exclude other causes of raised ICP only. Catheter venography was performed by an experienced interventional neuroradiologist under local anesthesia, via common femoral venous access with the patient supine. Contrast injected via a microcatheter in the superior sagittal sinus (SSS) and digital subtraction venography demonstrated anatomical sinus detail. Venous measurements were recorded at stereotypical locations in the SSS, torcula, transverse and sigmoid sinuses, internal jugular veins, superior vena cava, right atrium, and inferior vena cava. Stenoses were characteristically demonstrated at the transverse sigmoid sinus junction and a pressure gradient of $8 \mathrm{~mm} \mathrm{Hg}$ was considered significant. In cases in which stenoses with significant manometric gradients were present, the choice of subsequent DVSS or CSF diversion surgery was offered with appropriate consent and counseling. In all patients choosing DVSS, clinical reassessment and catheter venography and manometry measurements were repeated 3-4 months after treatment.

\section{Stent Placement}

All venous sinus stenting procedures were performed under general anesthesia and full intravenous heparinization by interventional neuroradiologists with more than 5 years of experience. A standard unit protocol was followed. A large flexible guide catheter was positioned in the affected sigmoid sinus (typically 0.088 -inch Neuronmax, Penumbra Inc.), and the stenosis crossed with a 0.027-inch microcatheter/0.014-inch microwire to obtain planning venography and measure pre-stenting venous pressures. The stent (typically 9-mm self-expanding carotid Wallstent, Boston Scientific) is deployed over a stiff 0.014-inch microwire (usually a Platinum Plus microwire) under biplane fluoroscopic imaging (Axiom Artis, Siemens). An immediate poststenting venogram confirmed satisfactory stent deployment and $500 \mathrm{mg}$ intravenous aspirin was given, with a subsequent lifelong prescription of $75 \mathrm{mg}$ oral aspirin.

\section{Statistical Analysis}

Data were processed using GraphPad Prism software (version 6.0c) and Microsoft Excel. Stenting manometric outcomes were compared with a paired 2-tailed t-test. A chi-square test was performed to determine if there was a significant difference in numbers of patients on various medications (acetazolamide, furosemide, and topiramate), and clinical and radiographic patency outcomes between the primary and secondary groups. An unpaired 2-tailed t-test compared age, SSS, and pressure gradient reductions between the 2 groups. Retreatment (equivalent to survival) was defined as number of days from stent insertion until the next intervention or end of the follow-up period. Kaplan-Meier curves were compared using the log-rank (Mantel-Cox) test. Differences in positive clinical outcomes (either resolution or improvement of signs and symptoms) between the primary and secondary groups were assessed 
using a Wilcoxon matched-pairs signed-rank test. A p value $<0.05$ was considered significant.

\section{Results \\ Demographics}

Between September 2010 and March 2016, 41 patients (2 men and 39 women) underwent DVSS and 3-4 month poststenting venography/manometry. The mean age of the entire study population was 35.7 years (range 19-55 years). The mean time for the follow-up venography/manometry was 101 days (range 91-120 days).

All patients had confirmed IIH and had previously undergone unsuccessful medical management. At the time of stent placement 23 patients were receiving $250 \mathrm{mg}$ of acetazolamide twice daily; 1 of these patients was also taking furosemide (20 mg once daily) and 10 were also receiving topiramate (100 mg once daily). One patient was taking topiramate only (100 mg once daily). One patient was receiving furosemide only ( $20 \mathrm{mg}$ once daily) due to acetazolamide tolerance. One patient was being treated with nocturnal continuous positive airway pressure (Table 1).

Twenty-six patients had DVSS as a primary procedure and 15 had DVSS as a secondary procedure (due to persistent signs and symptoms despite surgical revision). In the secondary group, 3 had a lumboperitoneal (LP) shunt, 7 had a ventriculoperitoneal (VP) shunt, 1 VP shunt was placed during a foramen magnum decompression, $1 \mathrm{VP}$ shunt was placed during a bifrontal cranioplasty, and 3 patients had failed LP shunts with secondary VP shunts (Table 1). Once stents were placed, patients in the secondary group did not have the shunt settings adjusted within the study's time period.

\section{Clinical Symptoms}

All 41 patients presented with headaches; 40 were followed up, and these headaches completely resolved in 7 patients, improved in 19 , remained the same severity in 13 , and worsened in 1 patient (Fig. 1A). Thirty-five patients reported associated blurred vision, which resolved in 8, improved in 16, remained the same in 10 , and worsened in 1 patient (Fig. 1B). Twenty-one patients had associated tinnitus, and 19 were followed up, in which it resolved in

TABLE 1. Study population demographics

\begin{tabular}{|c|c|c|c|c|}
\hline \multirow[b]{2}{*}{ Demographic } & \multirow[b]{2}{*}{ Total (\%) } & \multicolumn{2}{|c|}{ DVSS Procedure } & \multirow{2}{*}{$\begin{array}{c}p \\
\text { Value }\end{array}$} \\
\hline & & Primary (\%) & Secondary (\%) & \\
\hline No. of patients & 41 & 26 & 15 & \\
\hline $\mathrm{M} / \mathrm{F}$ & $2 / 39$ & $0 / 26$ & $2 / 13$ & 0.1 \\
\hline $\begin{array}{l}\text { Mean age } \pm \text { SD } \\
\quad(y r s)\end{array}$ & $35.7 \pm 9.23$ & $36.7 \pm 8.92$ & $34.1 \pm 9.86$ & 0.4 \\
\hline Acetazolamide & $23(56.1)$ & $16(61.5)$ & $7(46.7)$ & 0.5 \\
\hline Furosemide & $2(4.9)$ & $1(3.8)$ & $1(6.7)$ & 1.0 \\
\hline Topiramate & $11(26.8)$ & $6(23.1)$ & $5(33.3)$ & 0.5 \\
\hline LP shunt & $3(7.3)$ & NA & $3(20.0)$ & NA \\
\hline VP shunt & $9(22.0)$ & NA & $9(60.0)$ & NA \\
\hline LP \& VP shunts & $3(7.3)$ & NA & $3(20.0)$ & NA \\
\hline
\end{tabular}

$\mathrm{NA}=$ not applicable.
9, improved in 3 , and remained unchanged in 7 patients (Fig. 1C).

\section{Clinical Signs}

Thirty patients had papilledema; following stent insertion, this resolved in 12, improved in 7 , and remained stable in 11 individuals (Fig. 1D). In the positive outcome group, $13(68.4 \%)$ reported an improvement in their blurred vision (9 primary and 4 secondary).

Twenty had visual field defects; of the 18 with available follow-up data, 7 demonstrated complete resolution of the defect, 1 improved, and 10 remained stable after stent placement (Fig. 1E). In the patients with positive outcomes, 6 (75\%) reported improved blurred vision (5 primary and 1 secondary).

Twenty-seven patients had abnormal visual acuity prior to venous stent insertion, which resolved in 10, improved in 2 , remained stable in 10 , and worsened in 1 . Four patients were lost to follow-up (Fig. 1F). In the positive outcome group, 7 (58.3\%) reported blurred vision improvement (5 primary and 2 secondary).

Figure 2 graphically depicts patients who experienced a positive outcome in their clinical symptoms (Fig. 2A) and signs (Fig. 2B), either a resolution or improvement.

\section{Radiographic Outcome}

Twenty-six patients underwent stent placement as a primary procedure, of whom 21 had a sustained transstenotic gradient obliteration at 3-4 months. Two of these 21 patients developed a stenosis of their contralateral nonstented transverse sinuses. These were subsequently treated with stents and their trans-stenotic gradients were obliterated at the later 3-4-month follow-up venography and manometry follow-up (Fig. 3).

Three patients developed an ipsilateral radiographic stenosis distal to the DVSS, but none of these warranted re-stenting as there were no significant pressure gradients across the new stenoses. Two patients developed in-stent thrombosis, of whom 1 was treated successfully with intravenous unfractionated heparin and the other treated with a second stent procedure with gradient obliteration on follow-up venography 3-4 months after revision.

Of the 15 patients who underwent stent placement as a secondary procedure, 12 had radiographic stent patency and stenosis obliteration on 3-4-month follow-up venography. Three patients developed restenosis ipsilateral to the DVSS, of which 1 was distal and 2 were proximal to the stent. A significant pressure gradient was found across 1 of the proximal stenoses and a second stent procedure was performed. In the remaining 2 restenoses, no significant pressure gradient was found and further treatment was not indicated.

\section{Complications}

There were no procedural complications. Ipsilateral frontal headache, often severe and lasting for hours to days, is a common and previously well-described early phenomenon, believed to be caused by dural irritation immediately following stent placement. There were 2 cases of delayed in-stent thrombosis requiring readmission and 

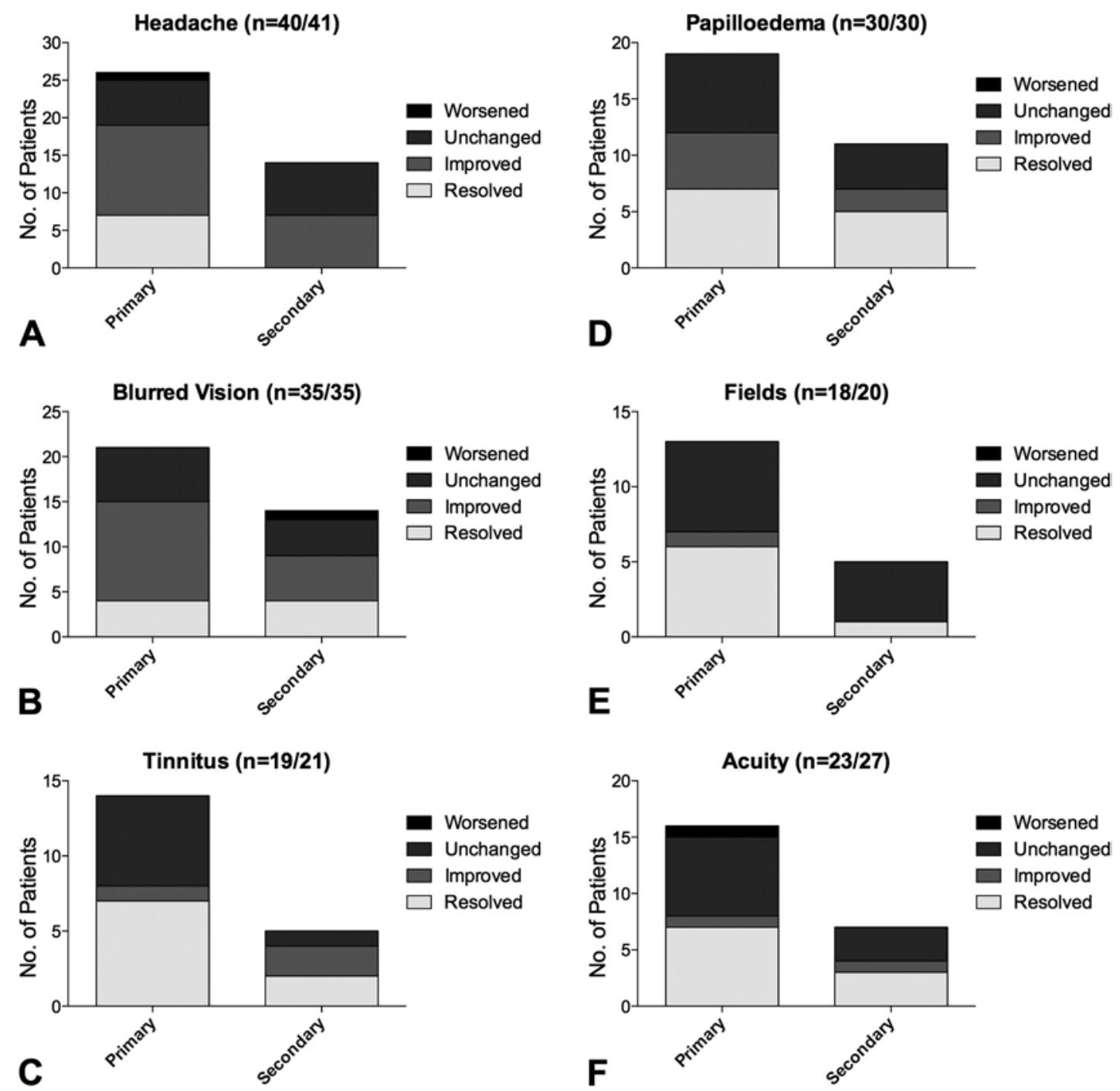

FIG. 1. Bar graphs showing changes in symptoms (A-C) and clinical signs (D-F) following DVSS in primary and secondary groups.

managed successfully with intravenous unfractionated heparin in 1 case and stent revision in the other. Three patients required early retreatments due to failed clinical response and manometrically significant stenotic recurrence outside the stent; 1 for ipsilateral proximal stenosis and 2 for contralateral stenoses.

\section{Pressure Measurements Before and After Stent Placement}

In all 41 patients, the mean $( \pm \mathrm{SD})$ pre-stent SSS pressure was $28.2 \pm 9.01 \mathrm{~mm} \mathrm{Hg}$. Mean SSS pressure at 3-4 months after stent placement was $17.5 \pm 6.94 \mathrm{~mm} \mathrm{Hg}$ (p $<0.0001$; Fig. 4A). Pre-stent pressure gradients across the stenoses were reduced from $17.5 \pm 8.01 \mathrm{~mm} \mathrm{Hg}$ to 6.17 $\pm 4.40 \mathrm{~mm} \mathrm{Hg} \mathrm{3-4} \mathrm{months} \mathrm{after} \mathrm{stent} \mathrm{placement}(\mathrm{p}<$ 0.0001; Fig. 4B).

In the primary group $(n=26)$, the mean pre-stent SSS pressure was $28.6 \pm 9.33 \mathrm{~mm} \mathrm{Hg}$. Mean SSS pressure at 3-4 months after stent placement was $16.8 \pm 7.38 \mathrm{~mm} \mathrm{Hg}$ ( $\mathrm{p}<0.0001$; Fig. 4C). Pre-stent pressure gradients across stenoses were reduced from $17.9 \pm 7.36 \mathrm{~mm} \mathrm{Hg}$ to 5.42 $\pm 4.17 \mathrm{~mm} \mathrm{Hg} \mathrm{3-4} \mathrm{months} \mathrm{after} \mathrm{stenting} \mathrm{(}<<0.0001$; Fig. 4D).

In the secondary group $(\mathrm{n}=15)$, the mean pre-stent SSS pressure was $27.6 \pm 8.71 \mathrm{~mm} \mathrm{Hg}$. The mean SSS pressure at 3-4 months after stent insertion was $17.8 \pm 4.40$ $\mathrm{mm} \mathrm{Hg}(\mathrm{p}<0.001$; Fig. 4E). Pre-stent pressure gradients across stenoses were reduced from $17.0 \pm 8.89 \mathrm{~mm} \mathrm{Hg}$ to $7.13 \pm 4.31 \mathrm{~mm} \mathrm{Hg} \mathrm{3-4} \mathrm{months} \mathrm{after} \mathrm{stenting} \mathrm{(}<0.001$; Fig. 4F).

\section{DVSS Retreatment Rate Analysis}

Stent "nonsurvival" or retreatment rate was therefore considered to have occurred where further intervention was required. Of the 5 patients requiring further surgical intervention, $1(3.85 \%)$ was in the primary group and $4(26.7 \%)$ were in the secondary group. Retreatment rate analysis revealed that, overall, $87.8 \%$ did not require further intervention at 120 days in 41 patients (Fig. 5A). In the primary group, this increased to $96.2 \%$ at 120 days in 26 patients. Those who underwent DVSS as a secondary 


\section{Symptom Improvement Comparison}

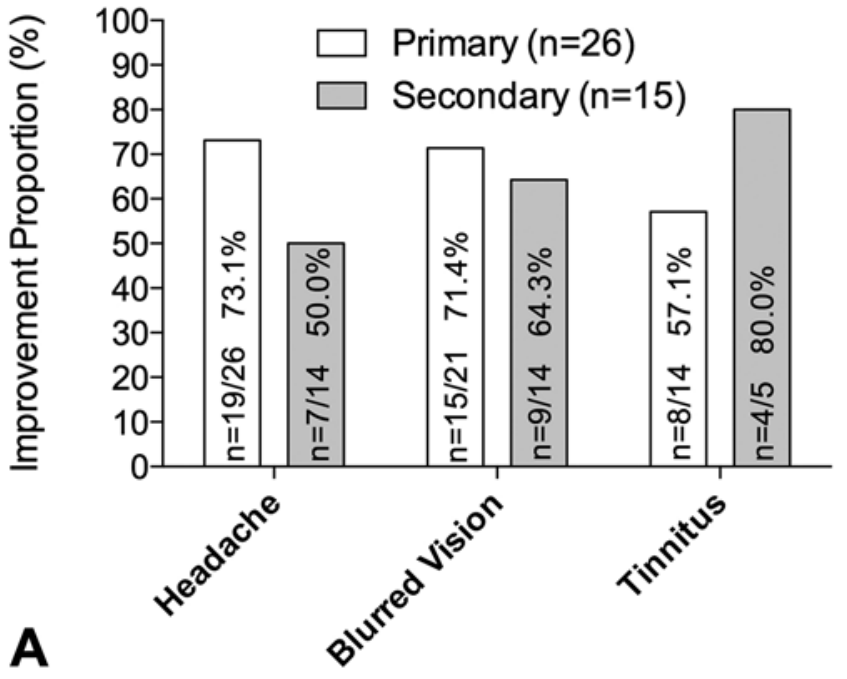

Sign Improvement Comparison

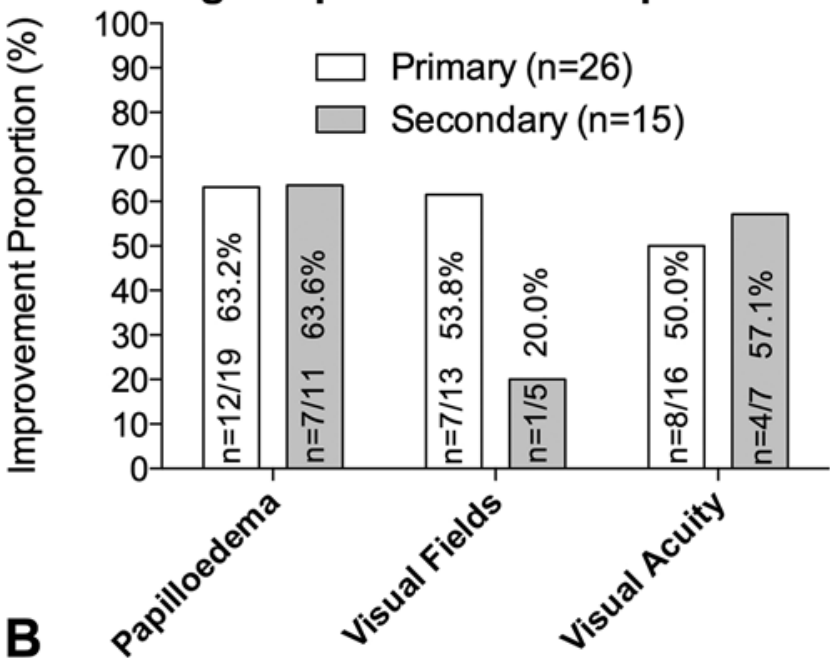

FIG. 2. Proportional representation of resolution or improvement in symptoms (A) and signs (B), comparing primary and secondary groups.

procedure had a $73.3 \%$ survival rate at 120 days in 15 patients. The log-rank (Mantel-Cox) test comparing retreatment rates between the primary and secondary groups found the difference to be significant ( $p=0.04$; Fig. 5B).

\section{Discussion}

This study reviewed radiographic, clinical, and manometric outcomes in patients who underwent DVSS as either a primary or secondary procedure to treat IIH. We also performed retreatment rate analysis of stents in the 2 groups of patients.

\section{Radiographic and Manometric Outcomes}

We found objective evidence of the effectiveness of DVSS in radiographic stenosis obliteration, 80.8\% (21 of 26 ) in the primary group versus $80.0 \%$ (12 of 15) in the secondary group. Therefore, DVSS is equally effective

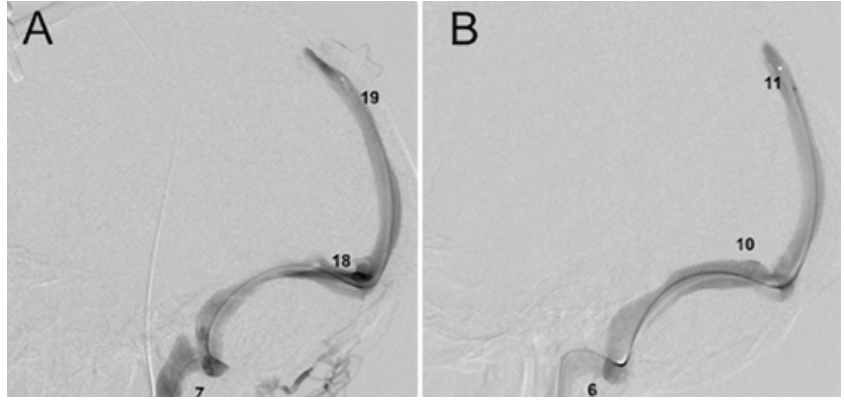

FIG. 3. A: Transverse sinus stenosis with a significant pressure gradient before stent insertion. B: Obliteration of the sinus stenosis and pressure gradient after stenting. The values represent pressure $(\mathrm{mm} \mathrm{Hg})$ measured at these locations.

regardless of whether it is a primary or secondary procedure.

Eight $(19.5 \%)$ of the 41 developed venographic restenosis after DVSS, 6 of which were stent-adjacent and 2 of which were contralateral stenosis at their nonstented transverse sinuses. However, only $5(12 \%)$ of 41 were manometrically significant ( 2 contralateral, 1 ipsilateral, and 2 in-stent thromboses) warranting retreatment; $4(9.8 \%)$ of 41 with stent insertion and $1(2.4 \%)$ of 41 with intravenous heparin.

Given the unpredictability of location and laterality of restenosis, the population of patients suffering restenosis after DVSS warrants further investigation to identify risk factors and stenosis mechanisms. High rates of restenosis following stent insertion has been previously reported and may be suggestive of an underlying pathophysiology of sinus stenosis or reflect the choice of stent technology. ${ }^{9,18,22}$ Patterns of restenosis in our patients conform to the hypothesis that raised ICP is the primary event in many patients with swollen brain parenchyma extrinsically compressing venous sinuses, with increasing venous hypertension and further increase in $\mathrm{ICP}^{4}$

Although there was a strong correlation between anatomical and manometric sinus findings in our data, it is the presence of a significant pressure gradient across the stenosis that defines a functionally significant stenosis, and this distinction is critical for appropriate patient selection and management. ${ }^{5}$ Our poststent pressure gradient reductions concur with those observed in a recent systematic review of 17 studies of 185 patients..$^{20}$ These investigators observed a reduction in pressure gradient of $15.7 \mathrm{~mm} \mathrm{Hg}$, not dissimilar to our overall reduction by $11.5 \mathrm{~mm} \mathrm{Hg}{ }^{20}$ Interestingly, in our study the primary group pressure gradient was reduced by $12.4 \mathrm{~mm} \mathrm{Hg}$, which was greater than the secondary group's decrease of $9.87 \mathrm{~mm} \mathrm{Hg}$, despite similar prestent pressure gradients of $17.9 \mathrm{~mm} \mathrm{Hg}$ and 17.0 $\mathrm{mm} \mathrm{Hg}$, respectively.

\section{Clinical Outcome}

Despite excellent radiographic and manometric outcomes, the clinical outcomes were modest with $65.0 \%$ of patients reporting a subjective improvement or resolution in their headache symptoms, and $63.3 \%$ having an objective improvement or resolution of papilledema on ophthalmological examination. It would be useful to confirm that 

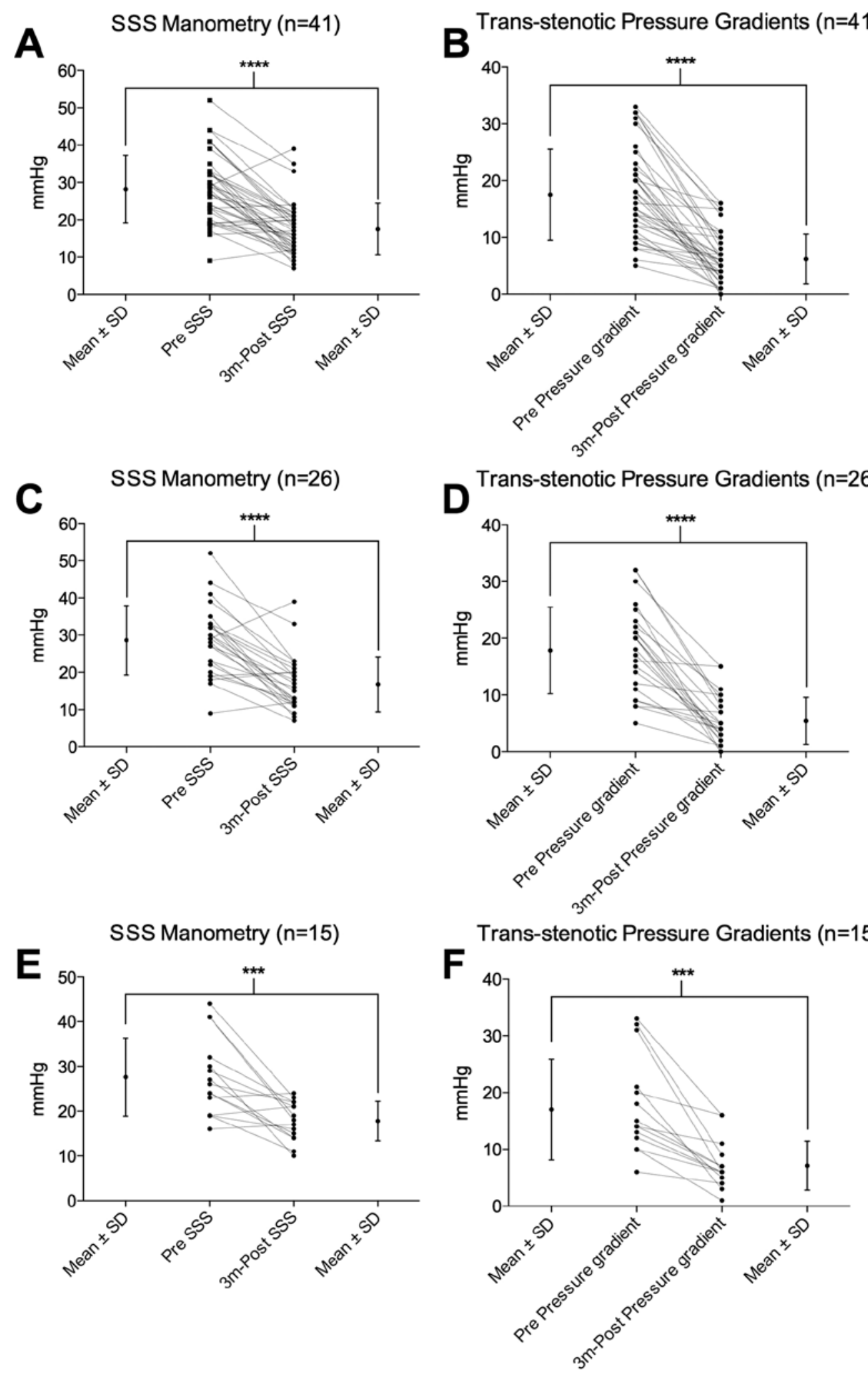

FIG. 4. Graphs of mean and individual patient results for SSS pressure. A and B: The total prestent SSS pressure of $28.2 \pm 9.01 \mathrm{~mm} H \mathrm{Hg}$ was reduced to $17.5 \pm 6.94 \mathrm{~mm} \mathrm{Hg} \mathrm{3-4} \mathrm{months}(3 \mathrm{~m})$ after stent placement $(\mathrm{p}<0.0001)$. The prestent pressure gradient was reduced from $17.5 \pm 8.01 \mathrm{~mm} \mathrm{Hg}$ to 6.17 $\pm 4.40 \mathrm{~mm} \mathrm{Hg} \mathrm{3-4} \mathrm{months} \mathrm{after} \mathrm{stenting} \mathrm{(} p<0.0001)$. C and D: The primary procedure prestent SSS pressure of $28.6 \pm 9.33 \mathrm{~mm} \mathrm{Hg}$ was reduced to $16.8 \pm 7.38 \mathrm{~mm} \mathrm{Hg}$ at $3-4$ months after stenting ( $p<0.0001)$. The prestent pressure gradient was reduced from $17.9 \pm 7.36 \mathrm{~mm} \mathrm{Hg}$ to $5.42 \pm 4.17 \mathrm{~mm}$ $\mathrm{Hg} 3-4$ months after stenting $(p<0.0001)$. E and F: The secondary procedure prestent SSS pressure of $27.6 \pm 8.71 \mathrm{~mm} \mathrm{Hg}$ was reduced to $17.8 \pm$ $4.40 \mathrm{~mm} \mathrm{Hg}$ at $3-4$ months after stent placement $(p<0.001)$. The prestent pressure gradient was reduced from $17.0 \pm 8.89 \mathrm{~mm} \mathrm{Hg}$ to $7.13 \pm 4.31 \mathrm{~mm}$ Hg 3-4 months after stenting $(p<0.001) .{ }^{* * *} p<0.0001,{ }^{* *} p<0.001$. 
symptomatic improvement correlated between objective and subjective measures to exclude placebo effect. However, our study design limited this and only papilledema was truly an objective measure, as the others required some degree of patient subjectivity.

Our finding that treatment of sinus stenosis does not universally result in improved visual symptoms has previously been reported. ${ }^{17}$ There was no significant difference in clinical outcome between those who had DVSS as a primary or secondary procedure (Table 2). However, other research groups have found better clinical outcomes, with 2 groups demonstrating functional improvements in symptoms and signs, including headache (81\%-88\%), papilledema $(90 \%-97 \%)$, visual symptoms $(87 \%)$, and tinnitus $(93 \%){ }^{14,22}$

To compare these figures with CSF diversion in IIH, Abubaker et al. demonstrated $89 \%$ and $80 \%$ postoperative symptom improvement rates after LP and VP shunting, respectively ( $\mathrm{n}=18$ for $\mathrm{LP}, \mathrm{n}=10$ for VP). ${ }^{1}$ Revision rates were $60 \%$ for LP shunts and $30 \%$ for VP shunts over an average follow-up of 4 years. ${ }^{1}$ Tarnaris et al. found that headaches improved in $71 \%$ and $60 \%$, and papilledema improved in $42 \%$ and $40 \%$, after LP and VP shunt placement, respectively (similar to DVSS outcomes in this study). Revision rates in this study were $40 \%$ for LP shunts and $22 \%$ for VP shunts $(n=24$ for LP shunts, $n=5$ for VP shunts) over a follow-up of 29 months. ${ }^{21}$

The discrepancy between radiological stenosis obliteration and clinical outcome may be explained by a number of factors. First, $68 \%$ of patients with IIH have a defined coexisting headache disorder such as migraine and tension-type headache, which would continue despite treatment of their raised ICP. ${ }^{6}$ Second, there may be distinct entities under the diagnosis of IIH that may need subclassification, i.e., DVSS-responsive and DVSS-unresponsive disease. The main contributor to raised ICP may be a focal intramural venous sinus stenosis in DVSS-responsive IIH and multifactorial in DVSS-unresponsive IIH.

\section{Stent Retreatment Analysis}

This is the first analysis comparing DVSS retreatment rates as primary and secondary procedures in IIH. The overall DVSS survival rate (defined by no requirement for a further procedure) was $87.8 \%$ at 120 days, which compares favorably to survival rates of VP shunts where failure rates range from $23 \%$ to $46.3 \%$, albeit over a longer time course..$^{8,16}$ The higher retreatment rates of stents inserted as a secondary procedure could be related to the fact that shunts change the CSF hydrodynamics interfering with stent function, or simply due to the fact that DVSS as a secondary procedure is performed in patients with a more aggressive or different form of IIH.

\section{Complications}

There were no deaths or permanent morbidities associated with DVSS in our cohort, although self-limiting ipsilateral frontal dural headache in the days following the procedure can be severe. Two patients $(4.9 \%)$ suffered delayed in-stent thrombus formation with recurrence of clinical symptoms, successfully treated using intravenous unfractionated heparin in 1 and DVSS revision in the other.
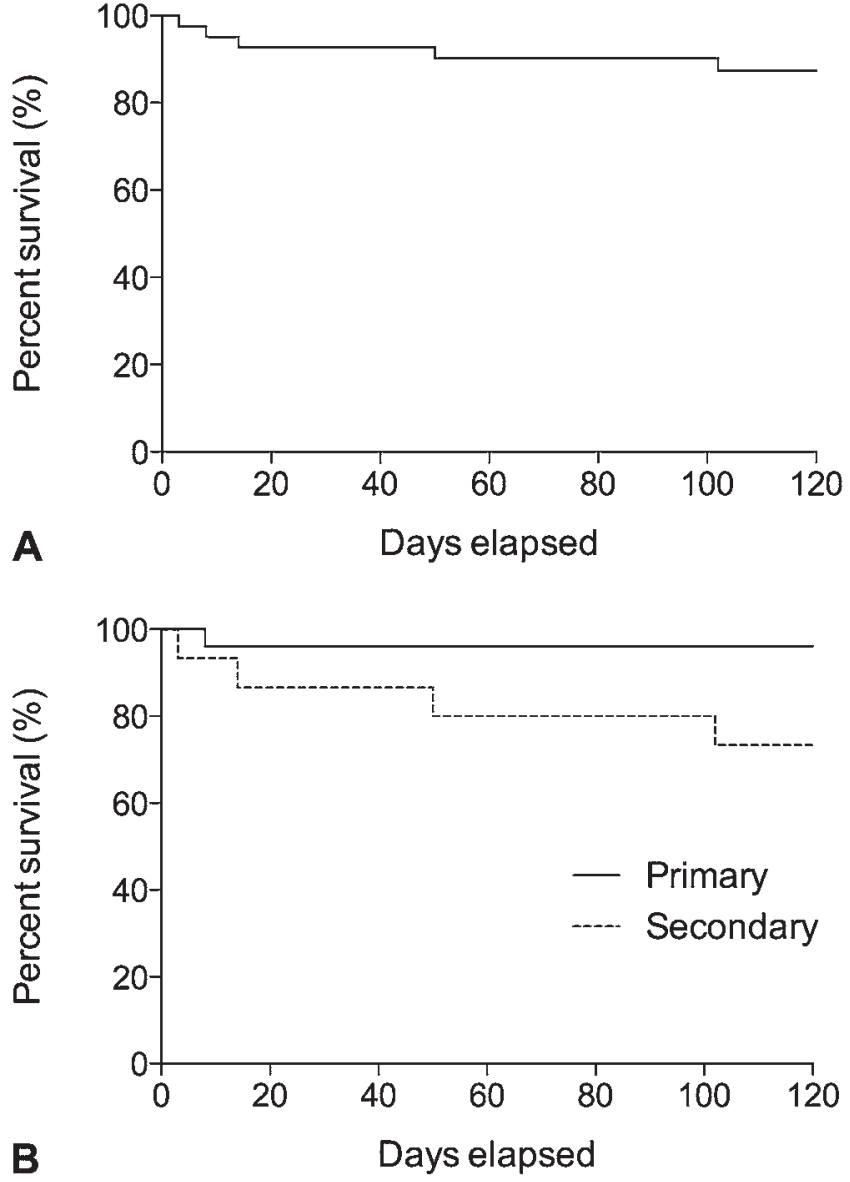

FIG. 5. Kaplan-Meier curves of the retreatment analysis for total stent retreatment rates $(\mathbf{A})$ and primary versus secondary retreatment rates $(\mathbf{B})$.

Further stenosis outside the stent requiring treatment occurred in $3(7.3 \%)$ of 41 , although such a "treatment failure" could be considered the natural history of the disease itself rather than a procedural complication. For comparison, a recent review by Puffer et al., reported a complication rate of $6 \%$ after DVSS. ${ }^{14}$ The complication rates observed after DVSS are significantly lower than those observed following VP shunts (47.1\%). ${ }^{11}$ However, while the complication rate is lower, the severity of complications is not negligible (venous sinus thrombosis is potentially catastrophic). One case series of 52 patients reported a subdural hemorrhage, a subarachnoid hemorrhage, and an intracerebral hemorrhage after DVSS. ${ }^{2}$

The authors concur with Ahmed et al. and Higgins et al. that DVSS only has a place in treating venous sinus stenosis with significant trans-stenotic pressure gradients..$^{2,7}$ However, the literature lacks Class 1 evidence demonstrating the superiority of DVSS over CSF diversion in IIH with focal venous sinus stenosis.

\section{Strengths and Limitations}

This is the first case series demonstrating radiographic and clinical outcome, pressure gradients, and survival of DVSS in those who underwent this procedure as a primary versus a secondary procedure. However, one limitation of 
TABLE 2. Clinical, radiographic, manometric, and survival comparisons between primary and secondary groups

\begin{tabular}{|c|c|c|c|c|}
\hline \multirow[b]{2}{*}{ Variable } & \multirow[b]{2}{*}{ Overall (\%) } & \multicolumn{2}{|c|}{ DVSS Procedure } & \multirow[b]{2}{*}{ p Value ${ }^{*}$} \\
\hline & & Primary (\%) & Secondary (\%) & \\
\hline Radiographic patency & $33 / 41(80.5)$ & $21 / 26(80.8)$ & $12 / 15(80.0)$ & 1.00 \\
\hline \multicolumn{5}{|l|}{ Resolution/improvement } \\
\hline Headache & $26 / 40 \dagger(65.0)$ & $19 / 26(73.1)$ & $7 / 14 \dagger(50.0)$ & 0.18 \\
\hline Blurred vision & $24 / 35(68.6)$ & $15 / 21(71.4)$ & $9 / 14(64.3)$ & 0.72 \\
\hline Tinnitus & $10 / 19 \ddagger(52.6)$ & $8 / 14 \dagger(57.1)$ & $4 / 5 \dagger(80.0)$ & 0.63 \\
\hline Papilledema & $19 / 30(63.3)$ & $12 / 19(63.2)$ & $7 / 11(63.6)$ & 1.00 \\
\hline Visual fields & $8 / 18 \ddagger(44.4)$ & $7 / 13 \dagger(53.8)$ & $1 / 5 \dagger(20.0)$ & 0.31 \\
\hline Visual acuity & $12 / 23 \rrbracket(52.2)$ & $8 / 16 \dagger(50.0)$ & $4 / 7 \S(57.1)$ & 1.00 \\
\hline Mean SSS pressure reduction \pm SD $(\mathrm{mm} \mathrm{Hg})$ & $11.1 \pm 8.79$ & $11.8 \pm 9.01$ & $9.80 \pm 8.55$ & 0.48 \\
\hline Mean pressure gradient reduction $\pm \mathrm{SD}(\mathrm{mm} \mathrm{Hg})$ & $11.5 \pm 7.47$ & $12.4 \pm 7.40$ & $9.87 \pm 7.58$ & 0.30 \\
\hline 120-day survival & $36(87.8)$ & $25(96.2)$ & $11(73.3)$ & 0.04 \\
\hline $\begin{array}{l}\text { Primary versus secondary. } \\
\text { One patient lost to follow-up. } \\
\text { Two patients lost to follow-up. } \\
\text { Three patients lost to follow-up. } \\
\text { Four patients lost to follow-up. }\end{array}$ & & & & \\
\hline
\end{tabular}

this study is that, as a case series, it has inherent selection bias and is, of course, nonrandomized and has no control cases. Second, although the follow-up duration is longer than other studies in the literature, it is still relatively short. Given the retrospective nature of the study, a considerable number of patients' objective clinical features were lost to follow-up. There is a paucity of evidence that directly correlates venous sinus pressure and ICP directly. We comment on SSS pressure, but this may not reflect ICP nor is it an appropriate surrogate marker. ${ }^{17}$ This may explain why the majority of patients appeared to have good radiographic outcome, but many symptoms and signs persisted. Despite this, we believe it was valuable to report the changing SSS pressure and its relationship to outcome.

\section{Future Research}

There are a number of points that require further research. First, studying the SSS pressure with an ICP monitor in situ would enable us to determine the nature of the relationship, if any, between SSS pressure and ICP. Second, more long-term data on outcomes, ICP, and SSS pressure are needed. At the time of writing this paper we are accumulating the data for 6- and 9-month outcomes. Third, the morphological appearance of the stenosis, and the effect of extrinsic versus intrinsic compression, is another important element that needs further research. Finally, this study suggests that complication rates of DVSS are lower than shunts. A prospective study with randomization is needed to compare DVSS and shunt insertion in IIH.

\section{Conclusions}

This study provides objective evidence of the effectiveness of venous sinus stent insertion in reducing venous sinus pressure 3-4 months after the procedure in the majority of patients with intracranial hypertension and focal venous sinus stenosis. Radiographic evidence of patent sinuses correlated with reduction in pressure gradients.
While our study suggests DVSS has lower complication rates than shunts, the outcome data are less clear. To definitively compare the outcomes between DVSS versus shunts in IIH, a randomized prospective study is needed.

\section{Key Points}

1. DVSS is equally effective in reducing clinical signs and symptoms of IIH, regardless of whether it is a primary or secondary procedure.

2. Only $63.3 \%$ of patients showed an improvement or resolution of papilledema.

3. Almost $20 \%$ of patients with IIH develop restenosis following DVSS.

4. The 3-4-month reduction in pressure gradient after stent placement is high, between 11 and $15 \mathrm{~mm} \mathrm{Hg}$.

5 . The pressure gradient reduction was greater in the primary group than the secondary group.

6. The DVSS survival rate was $87.8 \%$ at 120 days, which compares favorably to survival rates of VP shunts, in which failure rates range from $23 \%$ to $46.3 \%$.

7. Excluding restenosis, the complication rate after DVSS is $4.9 \%$.

8. There is no evidence demonstrating the superiority of DVSS over CSF diversion in IIH.

\section{References}

1. Abubaker K, Ali Z, Raza K, Bolger C, Rawluk D, O’Brien D: Idiopathic intracranial hypertension: lumboperitoneal shunts versus ventriculoperitoneal shunts-case series and literature review. Br J Neurosurg 25:94-99, 2011

2. Ahmed RM, Wilkinson M, Parker GD, Thurtell MJ, Macdonald J, McCluskey PJ, et al: Transverse sinus stenting for idiopathic intracranial hypertension: a review of 52 patients and of model predictions. AJNR Am J Neuroradiol 32:1408-1414, 2011

3. Baykan B, Ekizoğlu E, Altıkka Uzun G: An update on the pathophysiology of idiopathic intracranial hypertension alias pseudotumor cerebri. Agri 27:63-72, 2015

4. De Simone R, Ranieri A, Montella S, Bilo L, Cautiero F: 
The role of dural sinus stenosis in idiopathic intracranial hypertension pathogenesis: the self-limiting venous collapse feedback-loop model. Panminerva Med 56:201-209, 2014

5. Durst CR, Ornan DA, Reardon MA, Mehndiratta P, Mukherjee S, Starke RM, et al: Prevalence of dural venous sinus stenosis and hypoplasia in a generalized population. J Neurointerv Surg 8:1173-1177, 2016

6. Friedman DI, Rausch EA: Headache diagnoses in patients with treated idiopathic intracranial hypertension. Neurology 58:1551-1553, 2002

7. Higgins JN, Owler BK, Cousins C, Pickard JD: Venous sinus stenting for refractory benign intracranial hypertension. Lancet 359:228-230, 2002

8. Khan F, Rehman A, Shamim MS, Bari ME: Factors affecting ventriculoperitoneal shunt survival in adult patients. Surg Neurol Int 6:25, 2015

9. Kumpe DA, Bennett JL, Seinfeld J, Pelak VS, Chawla A, Tierney M: Dural sinus stent placement for idiopathic intracranial hypertension. J Neurosurg 116:538-548, 2012

10. Matloob SA, Toma AK, Thorne L, Watkins LD: Surgically managed idiopathic intracranial hypertension in adults: a single centre experience. Acta Neurochir (Wien) 157:20992103,2015

11. McGovern RA, Kelly KM, Chan AK, Morrissey NJ, McKhann GM II: Should ventriculoatrial shunting be the procedure of choice for normal-pressure hydrocephalus? J Neurosurg 120:1458-1464, 2014

12. Mollan SP, Ali F, Hassan-Smith G, Botfield H, Friedman DI, Sinclair AJ: Evolving evidence in adult idiopathic intracranial hypertension: pathophysiology and management. J Neurol Neurosurg Psychiatry 87:982-992, 2016

13. Piper RJ, Kalyvas AV, Young AM, Hughes MA, Jamjoom AA, Fouyas IP: Interventions for idiopathic intracranial hypertension. Cochrane Database Syst Rev 8:CD003434, 2015

14. Puffer RC, Mustafa W, Lanzino G: Venous sinus stenting for idiopathic intracranial hypertension: a review of the literature. J Neurointerv Surg 5:483-486, 2013

15. Radhakrishnan K, Ahlskog JE, Cross SA, Kurland LT, O'Fallon WM: Idiopathic intracranial hypertension (pseudotumor cerebri). Descriptive epidemiology in Rochester, Minn, 1976 to 1990. Arch Neurol 50:78-80, 1993

16. Reddy GK, Bollam P, Caldito G: Long-term outcomes of ventriculoperitoneal shunt surgery in patients with hydrocephalus. World Neurosurg 81:404-410, 2014

17. Riggeal BD, Bruce BB, Saindane AM, Ridha MA, Kelly LP, Newman NJ, et al: Clinical course of idiopathic intracranial hypertension with transverse sinus stenosis. Neurology 80:289-295, 2013

18. Rohr A, Dörner L, Stingele R, Buhl R, Alfke K, Jansen O: Reversibility of venous sinus obstruction in idiopathic intracranial hypertension. AJNR Am J Neuroradiol 28:656659,2007
19. Smith JL: Whence pseudotumor cerebri? J Clin Neuroophthalmol 5:55-56, 1985

20. Starke RM, Wang T, Ding D, Durst CR, Crowley RW, Chalouhi N, et al: Endovascular treatment of venous sinus stenosis in idiopathic intracranial hypertension: complications, neurological outcomes, and radiographic results. Sci World J 2015:140408, 2015

21. Tarnaris A, Toma AK, Watkins LD, Kitchen ND: Is there a difference in outcomes of patients with idiopathic intracranial hypertension with the choice of cerebrospinal fluid diversion site: a single centre experience. Clin Neurol Neurosurg 113:477-479, 2011

22. Teleb MS, Cziep ME, Issa M, Lazzaro M, Asif K, Hong SH, et al: Stenting and angioplasty for idiopathic intracranial hypertension: a case series with clinical, angiographic, ophthalmological, complication, and pressure reporting. J Neuroimaging 25:72-80, 2015

23. Xu K, Yu T, Yuan Y, Yu J: Current status of the application of intracranial venous sinus stenting. Int J Med Sci 12:780789,2015

\section{Disclosures}

Dr. Watkins reports honoraria and advisory board participation for Medtronic, St. Jude Medical, B Braun, and Codman.

\section{Author Contributions}

Conception and design: Asif, Craven, Toma. Acquisition of data: Asif, Craven, Shah. Analysis and interpretation of data: Asif, Craven. Drafting the article: Asif, Craven, Siddiqui. Critically revising the article: Asif, Craven, Robertson, Toma. Reviewed submitted version of manuscript: Asif, Craven, Siddiqui, Thorne, Robertson, Watkins, Toma. Approved the final version of the manuscript on behalf of all authors: Asif. Statistical analysis: Asif, Craven. Administrative/technical/material support: Matloob, Robertson. Study supervision: Thorne, Watkins, Toma.

\section{Supplemental Information}

\section{Previous Presentations}

Portions of this work were presented in abstract form at Hydrocephalus 2015, the 7th Annual Meeting of the International Society for Hydrocephalus and CSF Disorders, in Banff, Alberta, Canada, September 2015.

\section{Correspondence}

Hasan Asif, Victor Horsley Department of Neurosurgery, National Hospital for Neurology and Neurosurgery, Queen Square, London WC1N 3BG, United Kingdom. email: hasan.asif@doctors.org.uk. 